\title{
A new zebrafish model to study the link between TBI and dementia
}

Alyenbaawi, H. et al. Elife 10, e58744 (2021)

Traumatic brain injury (TBI) has been associated with an increased risk of different forms of dementia, including chronic traumatic encephalopathy (CTE), but the mechanisms linking the two pathologies are not fully understood. Many rodent models have been developed to replicate features and long-lasting deleterious effects of TBI, but those are impractical to study CNS changes in a living animal.

A new study in Elife describes the development of a larval zebrafish TBI model, which offers many technical advantages over rodent models including the fish's transparency. Using this TBI model in combination with a new tauopathy reporter line and a fluorescent reporter of neural activity, investigators led by Ted Allison from the University of Alberta identified a role for seizure activity in the prion-like spread of tauopathy following TBI.

The researchers induced TBI in the zebrafish larvae by loading them into a syringe with a closed valve stopper; applying a hit on the plunger then produces a pressure wave through the fish body, thereby mimicking the pressure or shock waves experienced during human blast injury. The injury was applied three times to the larvae to reduce technical variability. The larvae subjected to the traumatic injury paradigm presented with hemorrhages, blood flow abnormalities and increased cell death in the brain, which are characteristic features of human TBI. TBI larvae also exhibited a high propensity for spontaneous seizures, a frequent condition in patients with TBI caused by abnormal excessive neuronal excitability. Combining the TBI model with CaMPARI optogenetic methods confirmed a sharp increase of neuronal activity in the larvae during TBI.

The team also engineered a tauopathy biosensor transgenic zebrafish line that expresses a fluorescent human Tau reporter protein to detect Tau pathology in the zebrafish larvae. Combining the Tau biosensor with the TBI model revealed that TBI induces Tau aggregates, as measured by the number of GFP+ puncta in the brain and spinal cord of the larvae. Application of anticonvulsant drugs to reduce seizure activity and neuronal activity decreased the amount of GFP+ puncta in many larvae, suggesting that post-traumatic seizures link TBI to subsequent Tau pathology.

In conclusion, this study presents a new model that offers the ability to assess neural activity and Tau pathology in unrestrained organisms after TBI, and it identifies post-traumatic seizures as a potential druggable target to prevent the development of CTE following TBI.

Alexandra Le Bras

Published online: 16 February 2021

https://doi.org/10.1038/s41684-021-00728-9

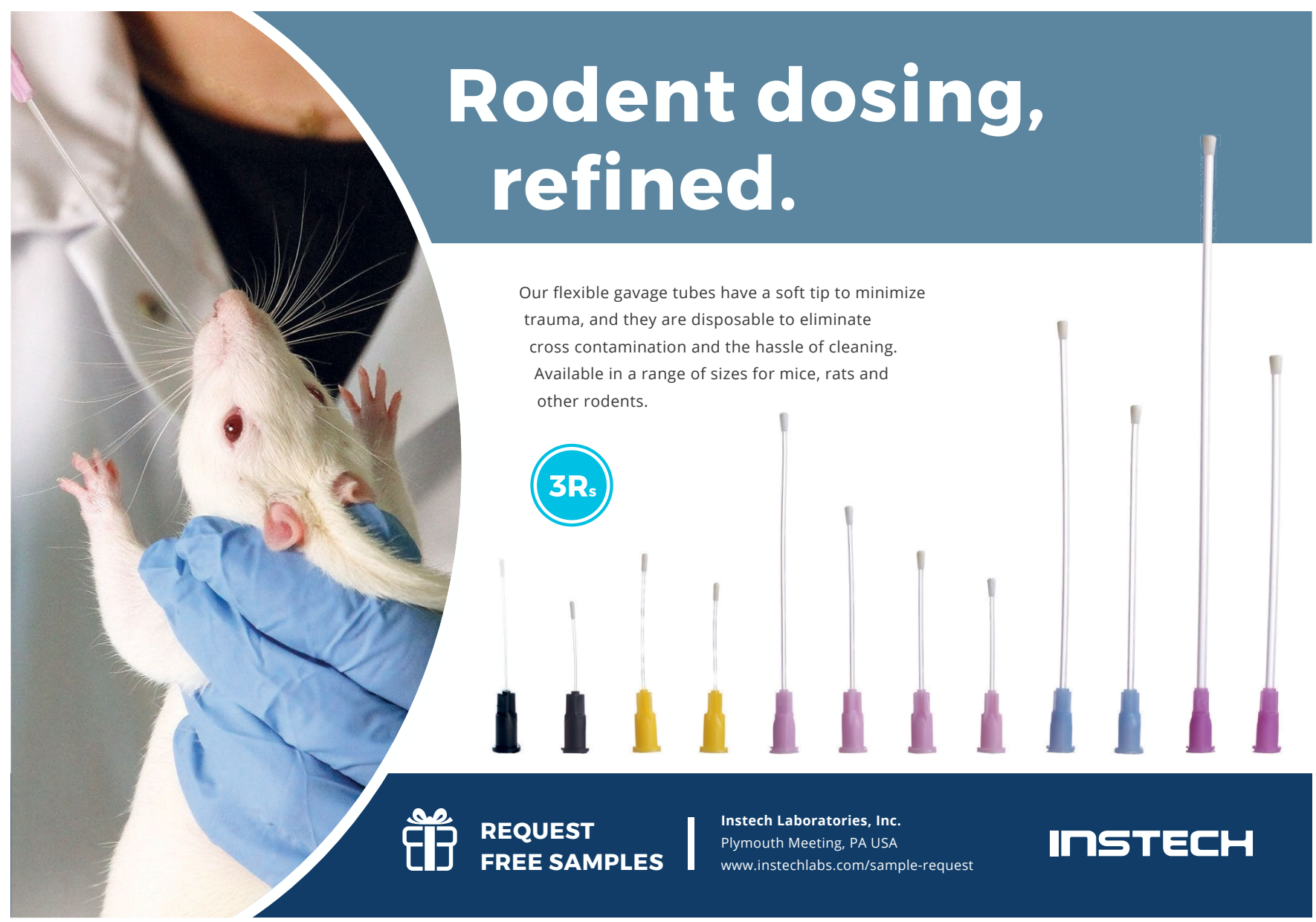

